\title{
Concevoir une formation en ligne à partir d'une étude sur les enjeux interculturels des ingénieurs
}

\section{Mélanie Le Forestier}

\section{(2) OpenEdition}

1 Journals

\section{Electronic version}

URL: http://journals.openedition.org/ripes/3085

DOI: 10.4000/ripes.3085

ISSN: 2076-8427

\section{Publisher}

Association internationale de pédagogie universitaire

\section{Electronic reference}

Mélanie Le Forestier, "Concevoir une formation en ligne à partir d'une étude sur les enjeux interculturels des ingénieurs", Revue internationale de pédagogie de l'enseignement supérieur [Online], 37(1) | 2021, Online since 15 February 2021, connection on 16 February 2021. URL: http:// journals.openedition.org/ripes/3085 ; DOI: https://doi.org/10.4000/ripes.3085

This text was automatically generated on 16 February 2021.

Article L.111-1 du Code de la propriété intellectuelle. 


\title{
Concevoir une formation en ligne à partir d'une étude sur les enjeux interculturels des ingénieurs
}

\author{
Mélanie Le Forestier
}

\section{Introduction}

Dans cet article, nous proposons de revenir sur le processus de conception d'une formation en ligne que nous avons été amenée à développer pour l'IDEFI DEFI Diversités, un projet inter-établissements co-piloté par des écoles d'ingénieur toulousaines et albigeoises ${ }^{1}$. Ce cours, intitulé POMI (Préparer et Optimiser sa Mobilité Internationale), a pour principal objectif pédagogique d'accompagner les élèvesingénieurs dans le développement d'une démarche interculturelle en s'appuyant sur leur projet de mobilité internationale.

\subsection{Un contexte d'internationalisation de l'enseignement supérieur}

Dans un contexte de mondialisation économique accélérée (Rosa, 2010), l'internationalisation est devenue un enjeu important pour l'enseignement supérieur, conjuguant à la fois des "considérations politiques, économiques, socioculturelles et académiques" (De Wit et al., 2015). Ce qu'on appelle internationalisation de l'enseignement supérieur (IES) renvoie « au processus d'intégration de cette dimension internationale dans l'enseignement, la recherche et les services administratifs de l'institution" (Knight, 1999, p.13). Dans sa manifestation la plus contemporaine (Huang, 2007, De Wit et Merkx, 2012), l'IES est un phénomène qui s'inscrit dans les processus de mondialisation des marchés à la fois comme réponse et comme agent de cette même mondialisation (Knight, 2003), dans la mesure où elle accompagne et participe à l'internationalisation des marchés de l'emploi et à la demande croissante 
des recruteurs de personnels et d'employés au profil multiculturel (Cosnefroy et al., 2020, p. 11).

Pour répondre aux enjeux liés à cet «environnement académique globalisé et concurrentiel » (Motard, 2017, p. 2), les établissements de l'enseignement supérieur, dont les écoles d'ingénieurs, ont mis en place des dispositifs d'enseignement visant à former des étudiants performants dans un contexte international et multiculturel. Cela s'inscrit dans un contexte largement incitatif puisque les recommandations des organismes institutionnels français (comme la Commission des Titres d'Ingénieurs CTI), européens (Conseil de l'Europe) et internationaux (UNESCO) s'inscrivent pleinement dans cette dynamique. Ces attentes institutionnelles et professionnelles en matière d'internationalisation ont contribué à l'émergence de formations à l'interculturel visant à former des «intellectuels interculturels » (Conseil de l'Europe, 2008). Dans l'enseignement supérieur, comme dans les formations professionnelles, l'objectif est le plus souvent de former à la communication interculturelle ou au management interculturel afin que les apprenants acquièrent des compétences interculturelles.

\section{2. À l'origine du projet : de la nécessité de former les élèves- ingénieurs à l'interculturalité}

4 Dans le cadre des écoles d'ingénieurs, cela s'inscrit dans une politique internationale des établissements qui fait partie des orientations stratégiques majeures étudiées par la CTI pour l'accréditation des écoles (CTI, 2020, p. 58). L'ouverture internationale et multiculturelle occupe une place centrale dans l'offre de formation, que ce soit à travers l'internationalisation à domicile (Beelen et Jones, 2015) avec l'« accueil des étudiants internationaux sur place, [l']élaboration de programmes d'études internationalisés et [l']intégration de dimensions internationales dans les activités d'enseignement, d'apprentissage et de recherche menées dans les établissements nationaux » (Huang, 2007, p. 52), ou que ce soit par la mobilité internationale des élèves-ingénieurs, des enseignants et des chercheurs. Cette expérience internationale est d'ailleurs obligatoire pour l'obtention du diplôme d'ingénieur et « doit permettre de fournir aux élèves-ingénieurs un apprentissage multiculturel, interculturel et linguistique nécessaire dans le contexte actuel d'internationalisation des entreprises et donc du recrutement des cadres » (CTI, 2020, p. 58).

5 Cependant, les chercheurs du champ de l'internationalisation de l'enseignement supérieur ont mis en évidence que les échanges entre les étudiants locaux et internationaux sur les campus s'avèrent souvent limités et l'immersion dans un contexte multiculturel n'est pas garante du développement des compétences interculturelles (Knight, 1999, De Wit et al., 2015). En effet, peu de dispositifs pédagogiques accompagnent ces mobilités entrantes ou sortantes.

\subsection{Question de recherche}

6 Partant de ce constant, notre mission au sein de l'IDEFI DEFI Diversités a pour objectif de pallier ce manque. La particularité de notre travail a été d'articuler ce projet de conception pédagogique à une recherche visant à identifier les besoins spécifiques des formations et du contexte professionnel des ingénieurs en matière d'interculturalité. Le 
travail dont nous rendons compte dans cet article s'appuie sur une approche méthodologique qui doit nous permettre de répondre à la question de recherche suivante: Quelle conceptualisation du développement interculturel permet de construire un dispositif de formation adapté aux enjeux interculturels rencontrés par les ingénieurs professionnels?

7 Nous commencerons par présenter notre cadre théorique sur l'interculturalité, ainsi que la méthodologie de recherche qui a permis la conceptualisation du modèle général de démarche interculturelle. Nous poursuivrons avec une présentation de la formation en ligne en soulignant la façon dont ce travail de recherche sur l'interculturalité a fortement contribué à la construction du scénario pédagogique de POMI.

\section{Une approche renouvelée de l'interculturalité}

8 Notre cadre théorique s'inscrit dans une approche renouvelée de l'interculturalité (Abdallah-Pretceille, 2003, Dervin, 2017, Jullien, 2016) qui se départit d'une approche culturaliste et essentialiste des relations interculturelles. Nous souhaitions aussi éviter une deuxième approche tout autant répandue, qualifiée de "janusienne " par Dervin (2017), qui affirme « haut et fort la labilité des cultures [mais qui continuent de tenir] des propos essentialistes, culturalistes, voire déterministes » (Debono et Dervin, 2012) par exemple, «Je ne veux pas généraliser, mais il faut reconnaître que les Français sont... ». De fait, cette deuxième approche a du mal à se détacher des tendances objectivantes et généralisantes de la première. Notre approche théorique s'inscrit dans un troisième et plus récent paradigme qui s'appuie sur un «modèle des références plurielles » (Pierre, 2017). Partant d'une perspective critique des précédents courants, cette approche constructiviste et interactionniste remet en question l'idée de culture comme " sujet " (Wikan, 2002), pour examiner les identités individuelles plurielles (Sen, 2006, Kaufmann, 2004) et « l'intention des individus quand ils font appel à la culture ou plus exactement à certains éléments culturels" (Abdallah-Pretceille, 2003, p. 20). Ce paradigme "se situe dans la question des co-constructions de soi, du "même" (individus du même pays) et de l'Autre » (Dervin, 2017, p. 90).

Ce dernier paradigme permet aussi de prendre des distances avec une conception de l'interculturel centrée sur la question des différences, des problèmes, des conflits, des « rencontres entre cultures» (Dervin, 2017, p. 37), ou encore sur la perception et la description des individus selon une seule particularité sociale ou culturelle et la supposée homogénéité des groupes d'appartenance ou de référence. Dans le prolongement des travaux d'Abdallah-Pretceille (2003) et de Dervin (2017) dans le champ de l'éducation interculturelle, il s'agit pour nous d'insister sur l'idée que les interactions interculturelles sont avant tout des interactions interpersonnelles où nous mettons en scène et négocions une pluralité de marqueurs identitaires comme «notre classe sociale, notre appartenance ethnique, notre sexe, notre profession, nos opinions politiques» (Sen, 2015, p. 27), ou encore «les visions du monde (worldviews) [et] les spécificités individuelles de chacun (émotions, sensibilités etc.) »(Dervin, 2017, p. 101). Notre approche de l'interculturalité s'appuie donc sur une définition qui insiste sur « la dimension processuelle, contextuelle et interactionnelle inhérentes aux relations dites "interculturelles" » (Lavanchy et al., 2011, p. 25) : «On entre alors dans la rencontre entre des individus pluriels, qui se co-construisent à travers/au-delà des identités solides et des différences culturelles apparentes, imaginées et/ou imposées, dans une 
historicité, une intersubjectivité et des contextes spécifiques d'interaction » (Lavanchy et al., 2011).

Le projet de recherche et celui d'ingénierie pédagogique se rejoignent ainsi dans l'objectif de ne pas se focaliser «sur la culture comme déterminant les comportements [mais plutôt de comprendre] ...la manière dont l'individu utilise les traits culturels pour dire et se dire, pour s'exprimer verbalement, corporellement, socialement, personnellement » (Abdallah-Pretceille, 2003, p. 20-21).

\subsection{L'identité collective et individuelle est plurielle}

11 Dans cette perspective, il est nécessaire de prendre en compte que l'hétérogénéité ne se situe pas uniquement à un niveau inter- ou intra-groupal, mais qu'elle est également individuelle. Nous souhaitons ici insister sur la « reconnaissance du caractère pluriel de notre identité » (Sen, 2015, p. 42), car la manière dont l'identité est perçue et définie impacte considérablement les interactions. L'identité, concept largement discuté dans le champ des sciences humaines et sociales dans la seconde moitié du XXe siècle, est un processus dynamique et relationnel (Erikson, 1968, Kaufmann, 2004, Kunnen et Bosma, 2006). Cette approche permet d'éviter trois principales erreurs communes commises dans une approche substantialiste de l'identité et qui reste de nos jours « la conception actuellement dominante » (Kaufmann, 2014). La première erreur consiste à « croire que l'identité renvoie à l'histoire, à notre mémoire, à nos racines " alors qu'en fait elle est « du côté de la subjectivité et de la production de sens » individuelle (Kaufmann, 2014, p. 22). La deuxième repose sur la confusion entre l'identité d'un individu et son « identité administrative, fondée sur des caractéristiques objectives de l'individu et la production du sens de sa vie » (Kaufmann, 2014). Si l'identification consiste à classer et finalement à figer l'identité des individus, la production de sens relève d'un travail subjectif labile et en constante évolution. En lien avec cette idée, la troisième erreur repose sur une définition de l'identité comme étant fixe, homogène et stable alors qu'il ne s'agit nullement d'une essence ou d'une substance à proprement parler (Kaufmann, 2014, p. 23).

Ne tenir compte que d'un critère unique définissant l'identité d'un individu est une erreur qu'on retrouve dans une approche qu'Amartya Sen définit comme "solitariste " (2015), c'est-à-dire que « les êtres humains sont envisagés comme les membres d'un seul et unique groupe" (Sen, 2015, p. 12). Or, aucune des catégories sociales et culturelles précédemment citées ne saurait définir une personne de manière exclusive et dans son entièreté. Repartant de l'approche théorique de notre recherche, il importe que notre projet pédagogique permette aux apprenants de réfléchir à la question du choix des individus dans la construction et l'affirmation de leur identité plurielle. C'est une composante essentielle dans l'apprentissage d'une démarche interculturelle.

Il est toutefois important de noter qu'un individu n'est pas « libre de tout, capable de choisir ses identifications " au-delà de toute contrainte externe (Dervin et Tournebise, 2012). L'environnement social et culturel et les expériences passées participent à la construction des cadres de référence des individus (Abdallah-Pretceille, 2003) et il s'agit d'éviter un «"zéro culturel", c'est-à-dire l'ignorance ou la négation de la dimension culturelle de la formation [comme un] "tout culturel", c'est-à-dire une survalorisation de la culture comme facteur déterminant les conduites et les apprentissages » (Abdallah-Pretceille, 2003, p. 10). 


\subsection{De l'utilité didactique de la notion de compétence interculturelle} Or, parler d'efficacité implique la nécessité et la capacité, de juger si ces individus communiquent effectivement de façon interculturellement efficace. La compétence interculturelle se doit donc d'être évaluable. Et pour cela, nous avons adapté le modèle de Michael Byram (1997) présenté dans le Tableau 1. Ce modèle s'appuie sur cinq composantes (savoirs, savoir-être, savoir-comprendre, savoir-faire et savoir-engager) qui correspondent chacune à des objectifs d'apprentissage et d'enseignement.

Tableau 1 : Tableau des facteurs de communication interculturelle (Byram, 1997, p. 34)

\begin{tabular}{|l|l|l|}
\hline & $\begin{array}{c}|c| \\
\text { Skills } \\
\text { Interpret and relate (savoir } \\
\text { comprendre) }\end{array}$ & \\
\hline $\begin{array}{l}\text { Knowledge } \\
\text { Of self and other; } \\
\text { Of interaction: } \\
\text { Individual and societal } \\
\text { (savoirs) }\end{array}$ & $\begin{array}{l}\text { Education } \\
\text { Political education } \\
\text { Critical cultural awareness } \\
\text { (savoir s'engager) }\end{array}$ & $\begin{array}{l}\text { Attitudes } \\
\text { Relativizing self } \\
\text { Valuing other } \\
\text { (savoir ettre) }\end{array}$ \\
\hline & $\begin{array}{c}\text { Skills } \\
\text { Discover and/or interact } \\
\text { (savoir apprendre/faire) }\end{array}$ & \\
\hline
\end{tabular}

Figure 2.1: Factors in intercultural communication (adapted from Byram 1997, p. 34).

Ce modèle a l'avantage de «fixer des objectifs d'enseignement-apprentissage plus ou moins précis à partir des différents types de savoirs " (Dervin, 2007, p. 105). Nous rejoignons toutefois la réserve de Dervin (2017) à l'égard de ce modèle, au sujet de la possibilité d'évaluer tous ces savoirs. Cela concerne plus particulièrement l'inclusion des savoir-être dans les référentiels de compétences et qui correspondent à des attitudes telles que la curiosité, l'ouverture d'esprit, ou encore la tolérance et 
l'honnêteté. Ces éléments, de l'ordre du jugement personnel et subjectif, sont difficilement évaluables par les enseignants, car ils renvoient aux dimensions affectives et émotionnelles des compétences (et non à la dimension cognitive, plus généralement évaluée). Qui pourraient, en effet, juger de l'honnêteté ou de l'authenticité dans une démarche de curiosité, d'ouverture d'esprit ou de tolérance? Dans notre projet, la compétence interculturelle est donc considérée, comme relevant plutôt « de l'ordre des savoir-faire que des savoirs » (Abdallah-Pretceille, 2003, p. 11).

\subsection{De la notion de compétence interculturelle à celle de démarche interculturelle}

19 Malgré ce premier cadrage, la notion de compétence interculturelle reste fortement ambigüe. En effet, comme le souligne à juste titre Dervin (2007), parler de compétence interculturelle, au singulier comme au pluriel, laisse sous-entendre l'idée, un peu paradoxale, qu'il existe une incompétence interculturelle. Or, il n'est pas simple de pouvoir juger, ni d'affirmer l'incompétence interculturelle d'une personne, si bien que nous avons travaillé sur une conceptualisation qui facilite l'observation des étapes d'une démarche interculturelle, présentée dans la section suivante.

\subsubsection{Définition d'une démarche interculturelle}

20 Afin de dépasser les limites de la notion de compétence interculturelle, nous lui avons préféré celle de démarche interculturelle, qui a aussi l'avantage de rendre compte du caractère dynamique de l'interculturel (Ogay, 2001). Nous concevons une démarche interculturelle comme relevant à la fois du champ de l'action et celui de la perception et associée à une motivation à agir et à des comportements. Elle est donc autant dans l'agir et le faire que dans l'être-au-monde (selon la phénoménologie de Merleau-Ponty [1976]). Nous proposons de la définir de la façon suivante : dans une interaction perçue comme interculturelle par les acteurs de cette interaction, une démarche interculturelle consiste à savoir mobiliser, de façon adaptée, ses compétences communicationnelles et sociales.

21 La démarche interculturelle s'inscrit dans le champ de la perception (Merleau-Ponty, 1976) et de la représentation sociale (Moscovici, 2003, Jodelet, 2003). En effet, nous souhaitons insister sur le fait qu'une situation interculturelle ne l'est pas par essence, elle le devient quand les acteurs d'une interaction se perçoivent comme appartenant à des groupes de référence différents et que cela implique des stratégies communicationnelles et comportementales visant à la réussite de cette interaction. Et si l'interculturalité relève de la perception en tant que relation au monde, elle ne peut se situer qu'en action, c'est-à-dire qu'elle relève aussi du champ de l'expérience (Rogers, 2005, Dewey, 2004).

\section{Méthodologie}

Comme notre objectif était l'identification des enjeux interculturels dans un contexte ingénieur, afin de construire un modèle de démarche interculturelle à même d'inspirer des dispositifs de formation adapté, nous nous sommes tout d'abord appuyée sur les résultats d'une étude de cas descriptive menée par notre collègue ${ }^{2}$, qui visait à faire un 
état des lieux des projets pédagogiques autour de l'interculturel dans les écoles de l'IDEFI. Cette étude préliminaire (étude 0) a été suivie par :

1. une recherche exploratoire qualitative (étude 1) que nous avons menée à partir d'entretiens courts avec des enseignants et des élèves-ingénieurs de l'INSA Toulouse et d'observations non participantes à des événements organisés autour de cette thématique et en lien avec le monde professionnel. L'objectif était de repérer la façon dont l'interculturalité était comprise et mobilisée dans un contexte ingénieur. 2. une étude autour d'entretiens compréhensifs longs (Kaufmann, 2011) menés avec huit ingénieurs en poste (étude 2), tous anciens élèves des écoles IDEFI, afin d'identifier les enjeux interculturels propres au contexte professionnel.

3. une étude quantitative auprès d'élèves-ingénieurs de l'INSA Toulouse (étude 3) visant à faire une analyse des besoins dans la préparation et le retour d'une mobilité internationale. La participation à cette enquête, à partir d'un questionnaire semidirectif en ligne, était uniquement sur la base du volontariat et aucune question n'était obligatoire. Cent soixante-trois étudiants, de différentes promotions et spécialités, ont accepté d'y répondre.

26 Les données des études 1 à 3 ont été étudiées à partir d'une analyse du discours (Maingueneau, 2014) et nous ont permis de conceptualiser un modèle général de démarche interculturelle qui reposait sur trois conditions :

27 - la nécessité de partir d'une définition élargie de l'interculturalité afin de prendre en compte les diverses diversités de chacun (Dervin, 2017) et de mettre en œuvre une déconstruction des stéréotypes et des biais implicites qui peuvent s'exprimer dans les discours. Les études $0,1,2$ ont en effet montré que la limite rencontrée dans les dispositifs pédagogiques étudiés est que les relations interculturelles s'inscrivent majoritairement dans le paradigme du "modèle des références nationales » (Pierre, 2017), avec l'utilisation récurrente de comparaisons et de "descriptions solides et statiques des "cultures" ou identités » (Pierre, 2017). Cette méthode a pour désavantage de figer le comportement et les réactions des individus à des traits qui seraient partagés par l'ensemble des membres d'un pays ou d'un groupe, au détriment de la personnalité et de l'identité plurielle de chaque individu.

28 - faire prendre conscience aux apprenants les mécanismes des interactions interculturelles et en particulier, de la dynamique communicationnelle, qui reposent sur: une mise en scène de chacun, une perception mutuelle et des variables d'identification mobilisées pour soi et les autres. Les résultats de l'étude 2 ont mis en évidence que les compétences mobilisées dans les situations interculturelles sont les mêmes que pour tous types d'interaction, mais elles sont spécifiquement utilisées comme stratégies pour réussir à communiquer ou collaborer avec des personnes perçues comme différentes, de par leur nationalité, leur métier, leur secteur d'activité professionnelle ou leur champ d'expertise.

29 - inviter les apprenants à penser la mobilité internationale en termes de compétences professionnelles, autant techniques qu'humaines et sociales. L'étude 3 a en effet révélé que les étudiants perçoivent leur mobilité comme une expérience de développement personnel et non comme une expérience professionnalisante.

Ce modèle est présenté dans la section suivante. 


\section{Conceptualisation d'un modèle général de démarche interculturelle}

Partant du cadre théorique et des résultats des études 0 à 3 , nous proposons de conceptualiser un modèle général de la démarche interculturelle adapté à un contexte ingénieur.

\subsection{Modèle général d'une démarche interculturelle}

Dans la définition de la démarche interculturelle, nous intégrons les enjeux spécifiques au contexte ingénieur identifiés dans l'enquête auprès des ingénieurs professionnels (étude 2). Cette recherche nous a permis d'élargir la notion d'interculturalité à des situations professionnelles dont l'objectif est, par définition, de produire quelque chose. La démarche interculturelle concerne donc aussi des activités professionnelles comme la gestion de projet et la conception et fabrication d'un produit ou de tout autre livrable. Comme nous l'avons précédemment indiqué, en tant que manière de faire, la démarche interculturelle est pleinement une démarche professionnelle et se retrouve à tous les niveaux dans la pratique professionnelle.

Figure 1 : Modèle général d'une démarche interculturelle adaptée au contexte ingénieur

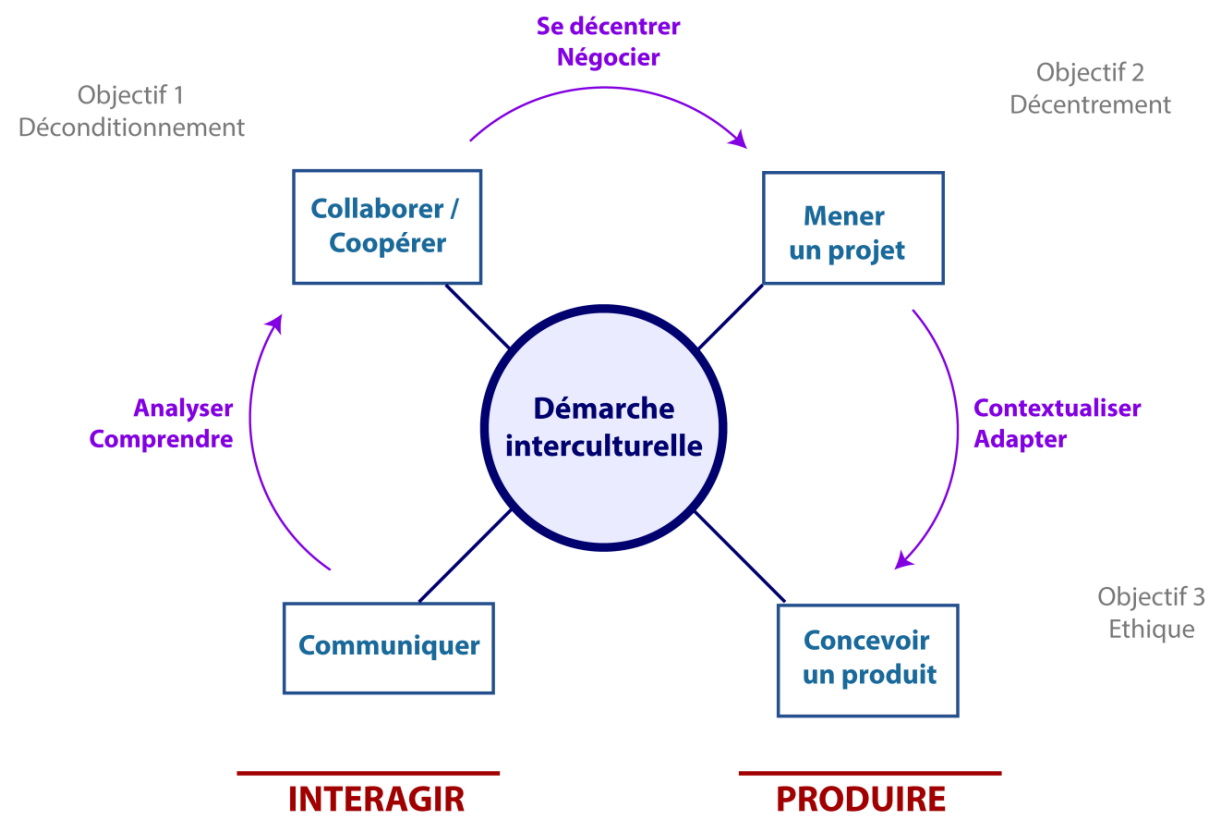

Comme l'illustre ce schéma (figure 1), une démarche interculturelle comporte plusieurs types de composantes qui se divisent, à un premier niveau, en deux grandes familles d'actions: des situations d'interaction d'un côté et des situations d'activités d'ingénierie de l'autre. À un deuxième niveau, chacune de ces familles comporte deux types de situations interculturelles. Pour les interactions, on trouve des situations de communication au sens général, directes ou indirectes, verbales ou non verbales, ainsi que des situations de collaboration ou coopération entre équipes, avec des partenaires, des collaborateurs ou encore avec des fournisseurs ou des clients. Cette première 
grande famille regroupe les situations habituellement prises en compte dans les recherches ou les formations interculturelles.

Partant de cette modélisation, il est alors possible d'inclure dans ce schéma les différents enjeux professionnels interculturels sous forme de six composantes (5 savoir-faire et un 1 savoir-agir). Dans le but de réussir à communiquer, collaborer ou coopérer, il est tout d'abord important d'analyser et de comprendre son environnement de travail, les représentations symboliques qui circulent, les comportements, les discours et les attitudes des interlocuteurs ainsi que de faire un travail réflexif sur ses propres actions et réactions. L'objectif principal d'une démarche interculturelle, dans ce contexte, est de se déconditionner (objectif 1), c'est-à-dire d'arriver à déconstruire et comprendre ses propres schémas de pensée ainsi que ceux des autres. Ces deux savoir-faire constituent une première étape nécessaire dans l'apprentissage interculturel.

Après avoir effectué ce premier travail analytique et réflexif, l'étape suivante, concernant plus particulièrement les situations de collaboration, de coopération et de gestion de projet, consiste à faire un travail de décentrement (savoir-agir) et de négociation (savoir-faire) afin de prendre en compte la pluralité des points de vue et des méthodes de travail. L'objectif de décentrement (objectif 2) permet de s'appuyer sur cette diversité pour co-construire une vision collective d'un projet.

Enfin, une troisième et dernière étape regroupe la capacité de contextualiser et de s'adapter aux besoins et aux attentes par rapport à un contexte et un public cible. Audelà des interactions communicationnelles et sociales entre personnes, il s'agit ici de mettre en œuvre les précédentes étapes de déconditionnement et de décentrement dans la gestion d'un projet et dans la conception et fabrication d'un produit, même dans le cadre d'un contexte industriel marqué par des enjeux de standardisation. Ces deux savoir-faire ouvrent la démarche interculturelle vers des considérations éthiques et humanistes en intégrant la question du sens de ce qui est produit (objectif 3 ).

\subsection{L'interculturalité en contexte ingénieur : une démarche professionnelle et éthique}

Jusqu'à présent, la démarche interculturelle a été principalement considérée à partir de sa dimension communicationnelle, c'est-à-dire dans le cadre d'interactions directes ou indirectes entre plusieurs individus ou groupes. Nous souhaitons désormais insister sur l'importance de l'hétérogénéité culturelle au sein même de la question scientifique et dans la façon d'appréhender l'ingénierie. L'accent mis sur « la pluralisation du monde et l'individualisation des choix » (Abdallah-Pretceille, 2020, p. 187) permet de révéler une pluralité de cultures scientifiques et techniques, autant dans la problématisation et la contextualisation des questions d'ingénierie, que dans la conception et la réalisation des solutions techniques apportées. Au-delà d'une base commune dans un même champ disciplinaire (telles des formules mathématiques ou de chimie par exemple), il existe de multiples façons de concevoir l'innovation technique. À titre d'exemple, nous pouvons citer les différents modèles alternatifs d'innovation que sont le low tech ou le wild tech (Grimaud et al., 2017) ou encore, pour prendre un sujet plus précis, la manière dont les processus d'automatisation et la robotique répondent à des enjeux sociétaux et idéologiques différents selon les contextes géographiques, économiques, politiques et 
sociaux (en guise d'exemple nous pouvons citer Stiegler (2015) pour le monde occidental et Robertson (2017) pour le Japon).

L'étude auprès des ingénieurs professionnels (étude 2) a aussi révélé que la question interculturelle est de nature éthique, ce que défendait déjà Martine Abdallah-Pretceille dans le champ de l'éducation (2003) et de la communication (2020). L'interculturalité met en effet en évidence que le fait de nommer, de classer dans des catégories sociales et culturelles ou des typologies, n'est pas un acte descriptif neutre, mais implique une considération éthique qui interroge ce constitue la norme (Abdallah-Pretceille 2020). De ce fait, la démarche interculturelle insiste sur l'importance de contextualiser ses actes, ses propos et sa pratique professionnelle dans un réseau d'intersubjectivités situés.

Pour le contexte ingénieur, la dimension éthique concerne aussi la manière de concevoir le métier d'ingénieur et «le sens de leur action dans le monde » (Chouteau et al., 2015). Dans la perspective d'un humanisme technique tel que pensé par les philosophes Gilbert Simondon et Gaston Berger, l'éthique interculturelle « renvoie à la nécessité de contextualiser les savoirs et de donner du sens aux actions humaines, à partir du rôle que chacun exerce dans la société, enseignant comme ingénieur " (Béchet et al., 2015, p. 2). Cela répond au « besoin de comprendre les implications de la science et la technique pour l'homme " (Béchet et al., 2015), selon des contextes variés et que permet le développement d'une "culture technique ", telle que l'a conçue par exemple G. Simondon (Guchet, 2015). Celle-ci renvoie au fait «que les techniques comprennent des dispositions culturelles, incluent des rapports sociaux, [et] impliquent des décisions politiques, économiques et sociales " (Chouteau et al., 2015).

Ce projet d'ingénierie pédagogique vise donc à prendre en compte cette dimension professionnelle et éthique afin de mettre en évidence l'intérêt pratique et professionnel de l'interculturalité pour les ingénieurs. Cela répond plus particulièrement à un objectif pédagogique autour de la transférabilité des apprentissages en articulant les compétences techniques et les compétences humaines et sociales. Tout l'enjeu est de réussir à intégrer les questions interculturelles dans les enseignements en sciences de l'ingénieur, ce que nous avons cherché à faire via le modèle de démarche culturelle dont nous proposons une application pédagogique ci-dessous.

\section{De la recherche à la conception pédagogique}

41 Cette conceptualisation de la démarche interculturelle nous a permis de construire un dispositif de formation en ligne à l'interculturalité adapté au contexte professionnel des ingénieurs. Nous cherchions par là à déconstruire un mythe bien établi qui repose sur l'idée que la mobilité internationale suffit à elle seule pour développer des compétences interculturelles (Knight, 2011). Il s'agissait donc de partir du projet de mobilité internationale obligatoire pour les élèves-ingénieurs, pour proposer une formation qui favorise un apprentissage interculturel.

\subsection{Transposition didactique dans une perspective fonctionnelle}

La première étape méthodologique dans la conception de cette formation en ligne a été de procéder à une transposition didactique de ce travail de recherche en objet d'enseignement. C'est bien une question de transposition puisque, comme le rappelle 
notamment Perrenoud (1998), cela implique une transformation et un changement partiel de la forme des savoirs scientifiques afin qu'ils deviennent des savoirs «"enseignables" et susceptibles d'être appris » (Perrenoud, 1998). Il s'agit ici de porter notre attention sur les objectifs pédagogiques de cette formation POMI qui doit permettre aux apprenants une mise en œuvre pratique de la démarche interculturelle dans le cadre de leurs expériences formatrices, puis professionnelles.

\subsubsection{Favoriser un apprentissage interculturel}

Le premier objectif pédagogique transversal de cette formation consiste à permettre aux apprenants de développer une démarche interculturelle, c'est-à-dire de mettre en pratique la conceptualisation théorique précédemment présentée. Notre travail de conception pédagogique vise donc à favoriser un apprentissage interculturel. Dans un modèle constructiviste et dynamique de l'apprentissage (Lebrun, 2007), nous concevons l'apprentissage interculturel comme la combinaison de plusieurs temps expérientiels qui s'appuie non seulement sur la «chaîne de transposition didactique " (Perrenoud, 1996), mais aussi sur le cycle de l'apprentissage expérientiel de Kolb (1984). En voici une proposition schématique articulant ces différents temps à la formation (comprise à la fois en tant qu'espace - la plateforme en ligne qui proposera le contenu de formation et en tant que temporalité - le suivi même de cette formation) :

1. Les connaissances et expériences préalables

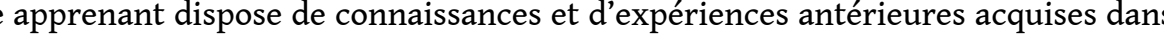
un environnement social et culturel particulier et de fait les "apprentissages impliquent que les connaissances préalables soient mobilisées et que les nouvelles soient réutilisées, fréquemment, dans des situations diverses » (Musial et al., 2011, p. 19).

2. Le temps pédagogique

Le suivi de la formation (individuel ou collectif, en autonomie ou en classe) entraîne la transformation des connaissances antérieures et la construction de connaissances nouvelles (Musial et al., 2011, p. 19), à travers un travail de déconstruction des biais et le développement d'une démarche interculturelle.

3. La mobilité internationale

Arrive ensuite le temps d'une nouvelle expérience qui permet la mise en œuvre pratique des connaissances acquises. Dans le cadre de POMI, il s'agit plus spécifiquement de la mobilité internationale sortante ou entrante des élèvesingénieurs.

4. Un temps réflexif

51 À la suite de cette nouvelle expérience vécue, ce cycle de l'apprentissage interculturel se conclut par un temps de réflexivité, individuelle ou collective, avec une mise en regard de cette nouvelle expérience par rapport aux expériences antérieures. Nous souhaitons insister sur l'importance de ce travail réflexif qui fait partie inhérente de la démarche interculturelle en ce qu'elle participe à son internalisation chez les apprenants ainsi qu'à la construction durable de nouvelles habitudes ${ }^{3}$. 


\subsubsection{Une approche pédagogique anti-biais} travail de déconstruction des biais de jugement implicites et explicites à travers une approche pédagogique anti-biais. C'est en effet une étape essentielle dans le développement d'une démarche interculturelle. Repartant de notre définition conceptuelle de cette démarche, cette formation vise à permettre aux apprenants de repérer et d'analyser les processus perceptifs dans le cadre d'interactions interculturelles, afin de déconstruire les stéréotypes, les préjugés et les biais de jugement et de valeur que les individus peuvent exprimer de manière implicite ou explicite, que ce soit dans le contexte de la formation d'ingénieur ou dans un contexte professionnel. Comme le précise Abdallah-Pretceille, l'objectif n'est «pas de s'arrêter sur les caractéristiques auto-attribuées ou hétéro-attribuées des autres, mais d'opérer, dans le même temps, un retour sur soi. [...] L'interrogation identitaire de soi par rapport à autrui fait partie intégrante de la démarche interculturelle » (AbdallahPretceille, 2003, p. 10).

Pour cela, il s'agit de proposer aux apprenants de repérer, pour mieux les écarter, les différents biais souvent associés à l'interculturel: "Le biais différentialiste (l'interculturel veut toujours dire différence de culture et de langue), le biais comparativiste (l'interculturel mène systématiquement à la comparaison des cultures, des valeurs, des habitudes etc.), le biais individualiste (c'est toujours la faute et la responsabilité de l'un des interlocuteurs alors qu'il partage cette responsabilité avec l'Autre), le biais problémiste ('interculturel fonctionne avant tout autour de problème, de communication, de « clash» des cultures et des valeurs)» (Dervin, 2017, p. 3-4).

Pour favoriser ce travail de déconstruction, nous privilégions notamment l'articulation de deux processus d'apprentissage complémentaires. Il s'agit, pour le premier, de la compréhension qui correspond à « l'interprétation (vraie ou fausse) que fait quelqu'un de quelque chose, à un moment donné, en fonction de ses connaissances antérieures (des concepts, des connaissances spécifiques de situation) mais aussi de ses souvenirs » (Musial et al. 2011, p. 20). Le second porte sur une prise de conscience qui est la « mise en œuvre par les activités d'analyse de ce que les apprenants produisent eux-mêmes, mais aussi dans l'analyse d'autres productions, notamment de productions qui ne respectent pas la règle qui est la cible de la prise de conscience " (Musial et al. 2011, p. 25).

\subsection{Scénarisation pédagogique à partir du modèle de démarche interculturelle}

Nous souhaitons désormais présenter concrètement le scénario pédagogique que nous avons conçu pour cette formation POMI. Ce travail de scénarisation s'est développé à partir du modèle général d'une démarche interculturelle (figure 1). Si ce modèle s'inscrit ici dans un contexte ingénieur, il est suffisamment général pour être transposé et adapté à une grande variété de situations professionnelles et de métiers. De même, cette formation s'appuie sur les projets de mobilité internationale des étudiants. Toutes les formes de mobilité sont prises en compte, entrante ou sortante, semestre d'étude, stage à l'étranger ou encore projet humanitaire.

Revue internationale de pédagogie de l'enseignement supérieur, 37(1) | 2021 


\subsubsection{Le cheminement pédagogique} approche méthodologique s'appuie sur une perspective fonctionnelle (en référence à la pédagogie fonctionnelle de John Dewey [Tsuin-Chen, 1958]) puisqu'il s'agit de répondre au contexte des formations d'ingénieur où l'approche par compétences est le modèle d'apprentissage préconisé par la CTI. Dans le cadre de ce nouveau paradigme, l'apprentissage est «centré sur l'appropriation de ces savoirs et sur leur insertion dans des problématiques pratiques » (Chauvigné et Coulet, 2010, p. 15). travailler les apprenants sur les trois grands objectifs d'apprentissage présentés dans le modèle, à partir de six modules (tableau 2) : les deux premiers modules répondent à l'objectif de déconditionnement ; les modules 3 et 4 visent l'objectif de décentrement ; enfin les modules 5 et 6 proposent de réfléchir à ce qu'est une démarche professionnelle interculturelle.

L'apprentissage interculturel se déroule en trois temps. Les quatre premiers modules préparent les apprenants à la mobilité internationale, à travers un travail de déconstruction des stéréotypes et d'une prise de conscience du caractère multiculturel de leur campus à domicile. S'en suit le temps de la mobilité même, où les apprenants tenteront de mettre en œuvre une démarche interculturelle (le temps de l'expérience). Puis au retour de leur mobilité, les étudiants sont invités à suivre les deux derniers modules qui visent, dans un travail réflexif, à penser leur mobilité comme une expérience professionnalisante. Ce dernier temps de la formation renvoie plus particulièrement à l'interculturalité en tant que démarche professionnelle et éthique.

Tableau 2. Modules et objectifs d'apprentissage de POMI

\begin{tabular}{|l|l|}
\hline Module 1 & $\begin{array}{l}\text { Quels enjeux interculturels pour les ingénieurs? } \\
\text { Introduction à la thématique interculturelle }\end{array}$ \\
\hline $\begin{array}{l}\text { Module } \\
2\end{array}$ & $\begin{array}{l}\text { Déconstruction des stéréotypes } \\
\text { J'apprends à repérer et déconstruire les biais implicites et explicites }\end{array}$ \\
\hline $\begin{array}{l}\text { Module } \\
\mathbf{3}\end{array}$ & $\begin{array}{l}\text { Mon campus à domicile } \\
\text { Je prends conscience du caractère multiculturel de mon campus }\end{array}$ \\
\hline $\begin{array}{l}\text { Module } \\
\text { Recherche d'informations } \\
\text { J'adopte une démarche proactive dans la préparation de mon séjour }\end{array}$ \\
\hline $\begin{array}{l}\text { Module } \\
5\end{array}$ & $\begin{array}{l}\text { Professionnalisation } 1 \\
\text { Je pense et valorise mon expérience en termes de compétences }\end{array}$ \\
\hline Module 6 & $\begin{array}{l}\text { Professionnalisation } 2 \\
\text { Je pense une question scientifique dans une perspective interculturelle }\end{array}$ \\
\hline
\end{tabular}

Revue internationale de pédagogie de l'enseignement supérieur, 37(1) | 2021 


\subsubsection{Compétences mobilisées pour chaque objectif d'apprentissage}

Afin de faire travailler les étudiants sur les trois objectifs d'une démarche interculturelle, six composantes à acquérir ont été identifiées et réparties dans les différents modules de formation (tableau 3). Nous nous sommes inspirée du modèle des compétences interculturelles proposées par Dervin (2007) qui comprend deux savoirfaire et un savoir-réagir/agir, correspondant aux trois premières composantes du tableau ci-dessous. Les trois composantes suivantes (savoir-faire 3 à 5) ont été pensées pour répondre aux besoins spécifiques identifiés dans le cadre de notre recherche. Dès lors que l'apprenant est en mesure de repérer et de déconstruire les représentations stéréotypées et biaisées, il lui est également nécessaire d'être capable de prendre en considération la pluralité des points de vue et des méthodes de travail (savoir-faire 3). C'est une compétence indispensable pour tout travail en équipe et dans la gestion de projet. Enfin, il est tout aussi important d'insister sur le rôle de la démarche interculturelle dans la pratique professionnelle des ingénieurs, afin de sortir d'une approche bien trop souvent technocentrée. Pour cela, le module 6 propose aux étudiants de travailler sur deux composantes qui leur permettront de penser la technique de façon contextualisée (savoir-faire 4) et de façon éthique (savoir-faire 5).

Tableau 3. Inventaire des compétences travaillées dans POMI

\begin{tabular}{|c|c|c|}
\hline $\begin{array}{l}\text { objectifs } \\
\text { d'apprentissage }\end{array}$ & Composantes à travailler & Modules concernés \\
\hline \multirow{2}{*}{$\begin{array}{ll}\text { 1. Se } \\
\text { déconditionner }\end{array}$} & $\begin{array}{l}\text { Savoir-faire } 1 \text { : discerner les } \\
\text { identifications }\end{array}$ & \multirow{2}{*}{$\begin{array}{l}\text { Modules } 1 \text { et } 2 \text { : Analyser et comprendre les } \\
\text { situations de communication et les } \\
\text { contextes, pour pouvoir collaborer ou } \\
\text { coopérer }\end{array}$} \\
\hline & $\begin{array}{l}\text { Savoir-faire } \quad 2: \quad \text { faire } \\
\text { attention aux discours }\end{array}$ & \\
\hline \multirow{2}{*}{ 2. Se décentrer } & $\begin{array}{l}\text { Savoir-réagir/agir } 1 \text { : } \\
\text { contrôler ses émotions et ses } \\
\text { discours }\end{array}$ & \multirow{2}{*}{$\begin{array}{l}\text { Modules } 3 \text { : aller à la rencontre des autres } \\
\text { Module } 4 \text { : se préparer au départ en } \\
\text { préparant une carte mentale sur le pays } \\
\text { choisi } \\
\text { Module } 5 \text { : analyse réflexive de la mobilité } \\
\text { en termes de compétences }\end{array}$} \\
\hline & $\begin{array}{l}\text { Savoir-faire } 3 \text { : prendre en } \\
\text { considération la pluralité des } \\
\text { points de vue }\end{array}$ & \\
\hline \multirow{2}{*}{$\begin{array}{l}\text { 3. Avoir une } \\
\text { démarche éthique }\end{array}$} & $\begin{array}{l}\text { Savoir-faire 4: penser la } \\
\text { technique } \\
\begin{array}{l}\text { de } \\
\text { contextualisée }\end{array}\end{array}$ & \multirow{2}{*}{$\begin{array}{l}\text { Modules 6: penser le métier d'ingénieur } \\
\text { dans une perspective interculturelle }\end{array}$} \\
\hline & $\begin{array}{l}\text { Savoir-faire 5: penser la } \\
\text { technique de façon éthique }\end{array}$ & \\
\hline
\end{tabular}

Ces six composantes sont présentées de façon détaillée aux apprenants dans le cadre du module 5. Celui-ci propose une activité de réflexivité sur la mobilité internationale en incitant les apprenants à penser leur expérience en termes de compétences. Pour cela, l'activité prend la forme d'un guide d'auto-évaluation que nous avons élaboré à partir

Revue internationale de pédagogie de l'enseignement supérieur, 37(1) | 2021 
du modèle des compétences interculturelles proposées par Dervin (2007) et que nous avons complété pour l'adapter à notre modèle d'une démarche interculturelle. Nous avons notamment repris la façon dont sont présentées les composantes dans le modèle de Dervin, car elle répond à un besoin que nous partageons. Il s'agit en effet de permettre aux apprenants de "vérifier si [leurs] propres actions/réactions/stratégies sont adéquates ou, le cas échéant, [de] se fixer des objectifs à remplir» (Dervin, 2007, p. 115).

61 Dans ce guide, chacune des six composantes est introduite par un paragraphe de cadrage, suivi de plusieurs items écrits à la première personne du singulier visant à faciliter le travail d'auto-évaluation (figure 2). Lorsque les apprenants considèrent un ou plusieurs items acquis, ils les cochent et rédigent un court paragraphe réflexif expliquant comment ils ont développé cette compétence. L'objectif est que les apprenants puissent se servir de ce guide tout au long de leur parcours de formation, au-delà de la seule mobilité internationale et qu'ils puissent y revenir pour le compléter en fonction de leurs diverses expériences interculturelles.

Figure 2. Extrait du guide d'auto-évaluation présentant les items pour le savoir-faire 3

\subsection{Savoir-faire III : prendre en considération la pluralité des points de vue}

J'ai conscience que dans le cadre d'un projet mené collectivement, je vais travailler avec des personnes ayant des points de vue différents du mien sur divers sujets. Mes interlocuteurs peuvent venir de secteurs d'activité, de spécialités, de disciplines scientifiques ou encore de métiers divers. Ils peuvent aborder un problème ou une question scientifique différemment que moi, avoir d'autres idées, ainsi que des méthodes de travail, qui leur sont personnelles, et pas nécessairement liées à une identité groupale ou nationale.

Dans le cas où ces situations sont traversées par des malentendus ou des discordes, j'essaye de ne pas considérer mon point de vue comme la meilleure solution (voire la seule !) pour résoudre un problème.

J'ai conscience que ce que je considère comme étant juste ne relève pas de la Vérité, mais d'un avis subjectif basé sur mes souvenirs et mes expériences, ou sur des informations qui $m$ 'ont été transmises (ex. : durant la formation, des activités associatives, stage, environnement familial et social...).

Je fais l'effort d'être à l'écoute des autres, sans tirer de conclusions hâtives, plutôt que d'insister sur la seule validité de mon raisonnement. J'ai la volonté de partager nos opinions respectives, et de travailler avec mes interlocuteurs pour trouver une solution collectivement.

\section{Conclusion : décloisonner l'interculturalité}

Dans cet article, nous avons souhaité montrer l'importance des enjeux interculturels pour la formation d'ingénieur à travers la conceptualisation d'un modèle de démarche interculturelle. Avec ce travail de définition et son rôle dans la conception pédagogique $\mathrm{du}$ cours POMI, il s'agissait pour nous de souligner le fait qu'« on ne peut enseigner directement des compétences, mais seulement créer les conditions de leur développement, au gré de dispositifs d'entraînement» (Perrenoud, 1998). Nous avons focalisé notre présentation sur l'articulation entre un travail de recherche, de modélisation de la démarche interculturelle et son application pédagogique. Une première phase de test de la plateforme en ligne, prévue au printemps 2021, devrait nous fournir des retours précieux sur les apports et les limites de cette approche. Néanmoins, nous pouvons d'ores et déjà observer que ce va-et-vient constant entre 
recherche et conception pédagogique constitue une véritable plus-value qui rend possible la création de contenus originaux adaptés aux spécificités du métier d'ingénieur, notamment pour cette thématique interculturelle.

Dans l'attente de ces premiers retours d'expérience, nous souhaitons conclure cet article sur l'idée qu'un décloisonnement de l'interculturalité dans l'enseignement supérieur est nécessaire. Dans les formations d'ingénieur, l'interculturel se retrouve traditionnellement dans des cours spécifiques comme les cours de langue, ou les cours en sciences humaines et sociales avec la communication interculturelle ou le management interculturel. Si ces enseignements participent à l'apprentissage interculturel des élèves-ingénieurs, ils restent trop souvent dans une approche culturaliste centrée sur un apprentissage de faits culturels. Sans remettre en question leur pertinence et leur efficacité, il est important de ne pas restreindre l'interculturalité à une question de contenu disciplinaire. L'interculturalité se construisant comme un champ de recherche pluridisciplinaire, nous souhaitons souligner l'importance d'adopter une approche transversale similaire dans l'enseignement. Ceci n'est d'ailleurs pas limité aux formations d'ingénieur et peut être transposé dans d'autres formations.

Un des enjeux de formation importants de cette transversalité, est d'assurer la transférabilité des apprentissages en décloisonnant les enseignements en sciences humaines et sociales et les cours de langue pour permettre aux étudiants de comprendre les articulations possibles entre ces cours et ceux de leur spécialité. C'est dans cette perspective que se situe notre définition d'une démarche interculturelle. Elle permet d'ouvrir les enjeux interculturels en les intégrant dans une approche transversale de l'ingénierie, autant du côté de la formulation d'un problème technique et scientifique, que du côté des réponses techniques proposées à des besoins spécifiques et contextualisés.

\section{Remerciements}

La recherche de Mélanie Le Forestier a été financée par l'IDEFI DEFI Diversités qui a bénéficié d'un financement dans le cadre du Programme d'Investissements d'Avenir (PIA) et une gestion opérationnelle de l'ANR.

\section{BIBLIOGRAPHY}

Abdallah-Pretceille, M. (2017). L'éducation interculturelle (5 édition). Presses Universitaires de France.

Abdallah-Pretceille, M. (2003). Former et éduquer en contexte hétérogène : Pour un humanisme du divers. Economica.

Abdallah-Pretceille, M. (2020). La communication interculturelle. Entre pertinence et impertinence. L'Harmattan. 
Bartel-Radic, A. (2009). La compétence interculturelle : état de l'art et perspectives. Management international, 13(4), 11-26. https://doi.org/10.7202/038582ar

Béchet, S., Escudié, M-P. et Jayet, Y. (2015). L’Institut Gaston Berger : «Sortez du troupeau ! ». Colloque pédagogique inter-INSA. http://colloque-pedagogie-2015.insa-rouen.fr/images/ programme/4eColPedago_AD1_04_ESCUDIE.pdf

Beelen, J. et Jones, E. (2015). Redefining Internationalization at Home. Dans A. Curaj, L. Matei, R. Pricopie et J. S. Salmi (dir), The European Higher Education Area, Between critical reflections and future policies. Springer Open.

Berardo, K. et Deardorff, D. K. (2012). Building cultural competence, Innovative Activities and Models. Stylus.

Byram, M. (1997). Teaching and Assessing Intercultural Communicative Competence. Multilingual Matters.

Chauvigné, C., Coulet, J-C. (2010). L'approche par compétences : un nouveau paradigme pour la pédagogie universitaire? Revue française de pédagogie, (172), 15-28. http:// www.journals.openedition.org/rfp/2169

Chouteau, M., Escudié, M.-P., Forest, J. et Nguyen, C. (2015). La technique est-elle condamnée à entrer par effraction dans notre culture? Phronesis, 4(2), 5-16. https://doi.org/10.7202/1033446ar Conseil de l'Europe. (2008). Livre blanc sur le dialogue interculturel.

Cosnefroy, L., De Ketele, J-M., Hugonnier, B., Palomba, D., Parmentier, P. et Uvalić-Trumbić, S. (2020). L'internationalisation de l'enseignement supérieur: Le meilleur des mondes? De Boeck Supérieur.

Debono, M. et Dervin, F. (2012). Pour une recherche interculturelle de «taille humaine »: entretien entre Fred Dervin \& Marc Debono. Dans C. Goï (éd.), Quelles recherches qualitatives en SHS. Approches interdisciplinaires de la diversité (p. 87-108.). L'Harmattan.

Dervin, F. (2007). Évaluer l'interculturel : problématiques et pistes de travail. Dans F. Dervin, et E. Suomela-Salmi (dir.), Évaluer les compétences langagières et interculturelles dans l'enseignement supérieur. Publication du Département d'études françaises, Université de Turku, (10), 96-122. Dervin, F. (2017). Compétences interculturelles. Éditions des archives contemporaines.

Dervin, F. et Tournebise, C. (2012). Rendez-vous ratés de l'interculturel en éducation? Une étude de cas de l'éducation à la communication interculturelle en Finlande. Semen, (34), 135-155. http:// journals.openedition.org/semen/9758

Dewey, J. (2004). L'école et l'enfant (édition de 1913, traduit par L. S. Pidoux). Fabert.

De Wit, H. et Merkx, G. (2012). The history of internationalisation of higher education. Dans D. Deardorff., H. De Wit, J. Heyl et T. Adams (éd.). The SAGE Handbook of International Higher Education. Sage Publications.

De Wit, H., Hunter, F., Howard, L. et Egron-Polak, E. (dir.). (2015). L'internationalisation de l'Enseignement Supérieur. Parlement Européen, Culture et Éducation.

Erikson, E. H. (1968). Identity, youth and crisis. Norton.

Faust, C. (2015). Représentations et gestion des compétences interculturelles. Le cas de Renault [thèse de doctorat, Université Paris-Est]. Université Paris-Est, Paris, France.

Grimaud, E., Tastevin, Y. P. et Vidal, D. (2017). Low tech, high tech, wild tech. Réinventer la technologie? Techniques \& Culture, (67), 12-29. https://journals.openedition.org/tc/8464 
Guchet, X. (2015). Pour un humanisme technologique. Culture, technique et société dans la philosophie de Gilbert Simondon. Presses Universitaires de France.

Huang, F. (2007). L'internationalisation de l'enseignement supérieur à l'ère de la mondialisation : ses répercussions en Chine et au Japon. Politiques et gestion de l'enseignement supérieur, 19(1), 49-64.

Jodelet, D. (dir). (2003). Les représentations sociales. Presses Universitaires de France.

Jullien, F. (2016). Il n'y a pas d'identité culturelle mais nous défendons les ressources d'une culture.

L'Herne.

Kaufmann, J-C. (2004). L'invention de soi. Une théorie de l'identité. Armand Colin.

Kaufmann, J-C. (2011). L'entretien compréhensif ( $3^{\mathrm{e}}$ édition). Armand Colin.

Kaufmann, J-C. (2014). Identités, la bombe à retardement. Textuel.

Knight, J. et De Wit, H. (éd.). (1999). Quality and internationalisation of higher education. OECD.

Knight, J. (2003). Interview de Jane Knight. IMHE Info, (1), 2.

Knight, J. (2011). Five Myths about Internationalization. International Higher Education. (62), 14-15.

Kolb, D. (2014). Experiential Learning: Experience as the Source of Learning and Development (2e éd.).

Pearson Education.

Kunnen, S. E. et Bosma, H. A. (2006). Le développement de l'identité : un processus relationnel et dynamique. L'orientation scolaire et professionnelle, 35(2). http://journals.openedition.org/osp/1061

Lavanchy, A., Gajardo, A. et Dervin, F. (dir.). (2011). Anthropologies de l'interculturalité.

L'Harmattan.

Lebrun, M. (2007). Théories et méthodes pédagogiques pour enseigner et apprendre : Quelle place pour les TIC dans l'éducation? De Boeck Supérieur.

Maingueneau, D. (2014). Discours et analyse du discours. Introduction. Armand Colin.

Merleau-Ponty, M. (1976). Phénoménologie de la perception (3éd.). Gallimard.

Moscovici, S. (2003). Des représentations collectives aux représentations sociales : éléments pour une histoire. Dans Jodelet, D. (dir), Les représentations sociales (p. 62-86). Presses Universitaires de France.

Motard, A-M. (2017). Les universités françaises et le défi de l'internationalisation au XXI ${ }^{e}$ siècle. Colloque international 21 défis du $21^{\mathrm{e}}$ siècle : sciences humaines et sociales. Université d'Etat Ilia, Tbilissi, Géorgie.

Musial, M., Pradère, F. et Tricot, A. (2011). Prendre en compte les apprentissages lors de la conception d'un scénario pédagogique. Recherche \& formation, 68, 15-30. https://doi.org/10.4000/ rechercheformation.1483

Ogay, T. (2001). De la compétence à la dynamique interculturelles. Peter Lang.

Perrenoud, P. (1996). Savoirs de référence, savoirs pratiques en formation des enseignants : une opposition discutable. Éducation et Recherche, (2), 234-250.

Perrenoud, P. (1998). La transposition didactique à partir de pratiques : des savoirs aux compétences. Revue des sciences de l'éducation, 24(3), 487-514.

Pierre, P. (2017). Trois courants de recherche en management interculturel dans les champs francophones. Dans P. Dupriez et B. Vanderlinden (dir), Au coeur de la dimension culturelle du management. L'Harmattan. 
Robertson, J. (2017). Robo sapiens japanicus: Robots, Gender, Family, and the Japanese Nation. University of California Press

Rogers, C. R. (2005). Le développement de la personne (traduit par E. L. Herbert). Dunod.

Rosa, H. (2010). Accélération: une critique sociale du temps (traduit par D. Renault). La Découverte.

Sen, A. (2015). Identité et violence : L'illusion du destin (traduit par S. Kleiman-Lafon). Odile Jacob.

Stiegler, B. (2015). La société automatique : Tome 1. L'avenir du travail. Fayard.

Tsuin-Chen, O. (1958). La doctrine pédagogique de John Dewey (2 éd.). Vrin.

Wikan, U. (2002). Generous Betrayal. Chicago University Press.

\section{NOTES}

1. Les écoles portant ce projet sont l'INP Toulouse, l'INSA Toulouse, l'ISAE Supaéro et l'IMT Mines Albi. Cet IDEFI propose un accompagnement des transformations pédagogiques afin de prendre en compte la diversité des profils et des parcours des étudiants.

2. Cet état des lieux a été mené par Lydia Bédouret, ingénieure pédagogique, et actuellement doctorante en psychologie cognitive des apprentissages à l'Université Toulouse 2 Jean Jaurès.

3. La question de l'internalisation et de la durabilité de ce nouvel habitus est développée dans le cadre de la thèse déjà citée de Lydia Bédouret, à partir d'une déconstruction des biais implicites.

\section{ABSTRACTS}

This article presents the design process of an online course, called POMI (Preparing and Optimizing your International Mobility). This course is for engineering students but can be adapted to fit other curricula. The main pedagogical objective is to guide students in the development of an intercultural approach using their experience of international mobility. The specificity of this project is to link this engineering pedagogy with a research project that aims to identify beforehand what are the specific intercultural needs of the engineering programs and of the professional engineering context. We will firstly introduce our theoretical framework that tries to move away from several methodological biases which remain prevailing in interdisciplinary studies on intercultural communication. We will broaden the definition of interculturality beyond the aspect of internationalization of higher education in order to also consider other intercultural training and professional contexts. Our theoretical model of an intercultural approach has played an important role in the design of this online course, which will be introduced in the second part of the article. Finally, we will introduce the pedagogy that has been used for this project, which is also the pedagogical approach we recommend to teachers who want to incorporate an intercultural dimension in their pedagogical practices.

Dans cet article, nous présenterons le processus de conception d'une formation en ligne appelée POMI (Préparer et Optimiser sa Mobilité Internationale) destinée à des élèves-ingénieurs, mais qui peut être transposée dans d'autres contextes de formation. Le principal objectif pédagogique est d'accompagner les apprenants dans le développement d'une démarche interculturelle en 
s'appuyant sur leur expérience de mobilité internationale, obligatoire en formation d'ingénieur. La particularité de ce projet a été d'articuler ce travail d'ingénierie pédagogique à une recherche visant à identifier, au préalable, les besoins spécifiques des formations d'ingénieur en matière d'interculturalité. Nous proposons donc une conceptualisation du développement interculturel dans un modèle de "démarche interculturelle" qui a permis de construire un dispositif de formation adapté aux enjeux interculturels rencontrés par les ingénieurs professionnels. En première partie d'article, nous préciserons la façon dont nous nous saisissons du champ de l'interculturalité et comment cela nous a permis de proposer une conceptualisation de ce que nous appelons une démarche interculturelle dans un contexte ingénieur. Nous montrerons ensuite comment le modèle de démarche interculturelle a servi à construire le scénario et le cheminement pédagogique du dispositif POMI. La formation étant toujours en cours de construction, nous conclurons l'article en présentant la pédagogie privilégiée et qui correspond à celle que nous proposons aux enseignants et responsables pédagogiques qui souhaitent intégrer la dimension interculturelle dans leur pratique enseignante.

\section{INDEX}

Mots-clés: conception pédagogique, recherche, transposition didactique, ingénieurs, interculturalité

\section{AUTHOR}

\section{MÉLANIE LE FORESTIER}

INSA Toulouse LERASS, Toulouse, France, leforest@insa-toulouse.fr 